\title{
DigITALCOMMONS
}

\section{A New Estimator for the Pickands Dependence Function}

Marta Ferreira

Centre of Mathematics of the University of Minho, Braga, Portugal, msferreira@math.uminho.pt

Follow this and additional works at: http://digitalcommons.wayne.edu/jmasm

Part of the Applied Statistics Commons, Social and Behavioral Sciences Commons, and the Statistical Theory Commons

\section{Recommended Citation}

Ferreira, M. (2017). A new estimator for the Pickands dependence function. Journal of Modern Applied Statistical Methods, 16(1), 350-363. doi: $10.22237 /$ jmasm/1493597940

This Regular Article is brought to you for free and open access by the Open Access Journals at DigitalCommons@WayneState. It has been accepted for inclusion in Journal of Modern Applied Statistical Methods by an authorized editor of DigitalCommons@WayneState. 


\section{A New Estimator for the Pickands Dependence Function}

\section{Cover Page Footnote}

The paper was partially supported by the Research Centre CEMAT through the Project UID/Multi/04621/

2013.

This regular article is available in Journal of Modern Applied Statistical Methods: http://digitalcommons.wayne.edu/jmasm/vol16/ 


\title{
A New Estimator for the Pickands Dependence Function
}

\author{
Marta Ferreira \\ Universidade do Minho \\ Braga, Portugal
}

The Pickands dependence function characterizes an extreme value copula, a useful tool in the modeling of multivariate extremes. A new estimator is presented along with its convergence properties and performance through simulation.

Keywords: $\quad$ Extreme value copula; tail dependence; nonparametric estimation

\section{Introduction}

Tail dependence is an important issue in several areas like finance, environment, engineering, among others, given the concern on the impact of the occurrence of joint extreme events. The copula concept provides a margin-free tool to describe the dependence structure of a random vector. Focusing on the bivariate case from now on, given a random pair $(X, Y)$ with joint distribution function $(\mathrm{df}) \mathrm{H}$, then it may be represented as

$$
\mathrm{H}(x, y)=\mathrm{C}(\mathrm{F}(x), \mathrm{G}(y))
$$

for all $\mathrm{x}, \mathrm{y} \in \mathbb{R}$, where $\mathrm{F}$ and $\mathrm{G}$ are the marginal df's of $X$ and $Y$, respectively. We always assume that $\mathrm{F}$ and $\mathrm{G}$ are continuous and thus copula $\mathrm{C}$ is unique (Sklar, 1959). Considering $U=\mathrm{F}(X)$ and $V=\mathrm{G}(Y)$, we may also write

$$
\mathrm{C}(u, v)=\mathrm{P}(U \leq u, V \leq v)
$$

for all $u, v \in[0,1]$. Extreme-value copulas arise in the limit of an increasing sample length of copulas of componentwise maxima of independent or strongly

Marta Ferreira is a researcher in the Centro de Matemática. Email them at: msferreira@math.uminho.pt. 


\section{MARTA FERREIRA}

mixing stationary sequences (Deheuvels, 1984; Hsing, 1989). Extreme-value copulas are completely determined by the Pickands dependence function, $\mathrm{A}:[0,1] \rightarrow[1 / 2,1]$, which is convex and satisfies $t \vee(1-t) \leq \mathrm{A}(t) \leq 1$, $\forall t \in[0,1]$, where $x \vee y=\max (x, y)$. More precisely, for all $0 \leq u, v \leq 1$,

$$
\mathrm{C}(u, v)=\exp \left(\log (u v) \mathrm{A}\left(\frac{\log (v)}{\log (u v)}\right)\right)
$$

Modeling applications of extreme-value copulas can be seen in Tawn (1988), Ghoudi, Khoudraji, and Rivest (1998), Frees and Valdez (1998), Coles, Heffernan, and Tawn (1999), Cebrian, Denuit, and Lambert (2003), McNeil, Frey, and Embrechts (2005), Salvadori, De Michele, Kottegoda, and Rosso (2007), amongst others. For instance, in volatile and bear markets, a dependence measure often used in lieu of Pearson's correlation to account for extreme events dependence is the so-called tail dependence coefficient (TDC) introduced in Sibuya (1960), usually denoted $\lambda$, which corresponds to $2(1-\mathrm{A}(0.5))$. The TDC ranges in $[0,1]$. The null boundary case corresponds to asymptotic tail independence, a very important topic in the statistics of extremes. Indeed, this case may not correspond to perfect independence but to a "residual" one that must be taken into account in order to avoid misleading risk estimates. See, e.g., Beirlant, Goegebeur, Segers, and Teugels (2004) and references therein.

Other representations than (1) may be considered, e.g., based on the stable tail dependence function, $l:[0, \infty)^{2} \rightarrow[0, \infty)$, which is convex, homogeneous of order one (i.e., $l(\alpha x, \alpha y)=\alpha l(x, y)$ for $\alpha>0$ ), satisfies $x \vee y \leq l(x, y) \leq x+y$, $\forall x, y \geq 0$, and $l(x, y)=(x+y) \mathrm{A}(y /(x+y))$, thus leading to

$$
C(u, v)=\exp (-l(-\log (u),-\log (v)))
$$

Representation (1) can also be formulated as

$$
\mathrm{C}\left(w^{1-t}, w^{t}\right)=w^{\mathrm{A}(t)}
$$

and thus, as well,

$$
\mathrm{C}\left(w^{1-t}, w^{t}\right)=w^{l(1-t, t)}
$$




\section{PICKANDS DEPENDENCE FUNCTION ESTIMATION}

Therefore, statistical inference on a bivariate extreme-value copula can be reduced to the estimation of a univariate Pickands dependence function (or a bivariate stable tail dependence function, although they are related).

Several parametric and non-parametric estimators of the Pickands dependence function are found in the literature. A wide survey on this topic is presented in Beirlant et al. (2004). Nonparametric estimation has been essentially based on the Pickands estimator (Pickands, 1981) and on the Capéraà-FougèresGenest (CFG) estimator (Capéraà, Fougères, \& Genest, 1997). Further modifications of the former can be seen in Deheuvels (1991) and Hall and Tajvidi (2000), while the latter can be found in Jiménez, Villa-Diharce, and Flores (2001), Zhang, Wells, and Peng (2008), and Gudendorf and Segers (2011); for both, see Segers (2007). All these approaches assume known margins, which is rather unrealistic in practice. Nonparametric versions of the Pickands and CFG estimators based on unknown margins are addressed in Abdous and Ghoudi (2005), Genest and Segers (2009), and Gudendorf and Segers (2012).

\section{Pickands Dependence Function: Estimators and Properties}

Let $(X, Y)$ be a random pair with joint $\mathrm{df} \mathrm{H}$ and continuous marginal $\mathrm{df}$ 's $\mathrm{F}$ and $\mathrm{G}$, respectively, such that, $U=\mathrm{F}(X)$ and $V=\mathrm{G}(Y)$. Let $\mathrm{C}$ be a bivariate extreme-value copula, i.e. of the form (1), characterizing the dependence between $X$ and $Y$. Thus $\mathrm{C}$ is the df of the random pair $(U, V)$.

Consider $S=-\log (U), T=-\log (V)$ and

$$
\xi(0)=\frac{S}{1-t} \wedge \frac{T}{t}, 0<t<1
$$

with $\xi(0)=S$ and $\xi(1)=T$. The random variables (rv's) $S$ and $T$ are Exponential with unit mean value and $\xi(t)$ is also exponentially distributed with mean values

$$
\mathrm{E}(\xi(t))=\frac{1}{\mathrm{~A}(t)} \quad \text { and } \quad \mathrm{E}(\log (\xi(t)))=-\log (\mathrm{A}(t))-\gamma
$$

where $\gamma$ denotes the Euler's constant $\int_{0}^{\infty} \log (x) \mathrm{e}^{-x} d x \approx 0.577$. These relations are the bases of, respectively, the Pickands and the CFG estimators by considering the empirical counterparts. More precisely, for a random sample $\left(X_{1}, Y_{1}\right), \ldots,\left(X_{n}, Y_{n}\right)$ 


\section{MARTA FERREIRA}

distributed as $(X, Y)$ such that $U_{i}=\mathrm{F}\left(X_{i}\right)$ and $V_{i}=\mathrm{G}\left(Y_{i}\right), S_{i}=-\log \left(U_{i}\right)=\xi_{i}(0)$, $T_{i}=-\log \left(V_{i}\right)=\xi_{i}(1)$ for all $i=1, \ldots, n$, with

$$
\xi_{i}(t)=\frac{S_{i}}{1-t} \wedge \frac{T_{i}}{t}, 0<t<1
$$

we have

$$
\frac{1}{\mathrm{~A}_{n}^{\mathrm{P}}(t)}=\frac{1}{n} \sum_{i=1}^{n} \xi_{i}(t)
$$

and

$$
\log \left(\mathrm{A}_{n}^{\mathrm{CFG}}(t)\right)=-\gamma-\frac{1}{n} \sum_{i=1}^{n} \log \left(\xi_{i}(t)\right)
$$

Whenever the margins $\mathrm{F}$ and $\mathrm{G}$ are unknown, the natural approach is to consider the respective marginal empirical $\mathrm{df}^{\prime} \mathrm{F}_{n}$ and $\mathrm{G}_{n}$ and take

$$
\hat{U}_{i}=\frac{n \mathrm{~F}_{n}\left(X_{i}\right)}{n+1}=\frac{1}{n+1} \sum_{j=1}^{n} \mathbb{I}_{\left\{X_{j} \leq X_{i}\right\}} \quad \text { and } \quad \hat{V}_{i}=\frac{n \mathrm{G}_{n}\left(Y_{i}\right)}{n+1}=\frac{1}{n+1} \sum_{j=1}^{n} \mathbb{I}_{\left\{Y_{j} \leq Y_{i}\right\}}
$$

where II is the indicator function. The replacement of $U_{i}$ and $V_{i}$ everywhere in the expressions above by, respectively, $\hat{U}_{i}$ and $\hat{V}_{i}$, leads now to

$$
\frac{1}{\hat{\mathrm{A}}_{n}^{\mathrm{P}}(t)}=\frac{1}{n} \sum_{i=1}^{n} \hat{\xi}_{i}(t)
$$

and

$$
\log \left(\hat{\mathrm{A}}_{n}^{\mathrm{CFG}}(t)\right)=-\gamma-\frac{1}{n} \sum_{i=1}^{n} \log \left(\hat{\xi}_{i}(t)\right)
$$

In order to satisfy the endpoint constraints $\mathrm{A}(0)=\mathrm{A}(1)=1$, endpoint corrected versions were considered, namely, 


\section{PICKANDS DEPENDENCE FUNCTION ESTIMATION}

$$
\frac{1}{\mathrm{~A}_{n, c}^{\mathrm{P}}(t)}=\frac{1}{\mathrm{~A}_{n}^{\mathrm{P}}(t)}-(1-t)\left(\frac{1}{\mathrm{~A}_{n}^{\mathrm{P}}(0)}-1\right)-t\left(\frac{1}{\mathrm{~A}_{n}^{\mathrm{P}}(1)}-1\right)
$$

and

$$
\log \left(\mathrm{A}_{n, c}^{\mathrm{CFG}}(t)\right)=\log \left(\mathrm{A}_{n}^{\mathrm{CFG}}(t)\right)-(1-t) \log \left(\mathrm{A}_{n}^{\mathrm{CFG}}(0)\right)-t \log \left(\mathrm{A}_{n}^{\mathrm{CFG}}(1)\right)
$$

Further developments on this topic can be found in Segers (2007). Similar procedures can be applied to the case of unknown marginal estimators and thus derive $\hat{\mathrm{A}}_{n, c}^{\mathrm{P}}(t)$ and $\hat{\mathrm{A}}_{n, c}^{\mathrm{CFG}}(t)$, although they are asymptotically equivalent to the respective uncorrected $\hat{\mathrm{A}}_{n}^{\mathrm{P}}(t)$ and $\hat{\mathrm{A}}_{n}^{\mathrm{CFG}}(t)$, as shown in Genest and Segers (2009). Another correction of the Pickands estimator based on Hall and Tajvidi (2000) is to consider

$$
\frac{1}{\hat{\mathrm{A}}_{n}^{\mathrm{HT}}(t)}=\frac{1}{n} \sum_{i=1}^{n} \bar{\xi}_{i}(t)
$$

with

$$
\bar{\xi}_{i}(t)=\frac{\bar{S}_{i}}{1-t} \wedge \frac{\bar{T}_{i}}{t}
$$

where $\bar{S}_{i}=n \hat{S}_{i} /\left(\hat{S}_{1}+\ldots+\hat{S}_{n}\right)$ and $\bar{T}_{i}=n \hat{T}_{i} /\left(\hat{T}_{1}+\ldots+\hat{T}_{n}\right), \hat{S}_{i}=-\log \left(\hat{U}_{i}\right)=\hat{\xi}_{i}(0)$, $\hat{T}_{i}=-\log \left(\hat{V}_{i}\right)=\hat{\xi}_{i}(1), i=1, \ldots, n$. We have $\hat{\mathrm{A}}_{n}^{\mathrm{HT}}(0)=\hat{\mathrm{A}}_{n}^{\mathrm{HT}}(1)=1$ and also $\hat{\mathrm{A}}_{n}^{\mathrm{HT}}(t) \geq t \vee(1-t)$ for all $0 \leq t \leq 1$. Relation $\hat{\mathrm{A}}_{n}^{\mathrm{HT}}(t)=\hat{\mathrm{A}}_{n}^{\mathrm{P}}(t) / \hat{\mathrm{A}}_{n}^{\mathrm{P}}(0)$ means that $\hat{\mathrm{A}}_{n}^{\mathrm{HT}}(t)$ and $\hat{\mathrm{A}}_{n}^{\mathrm{P}}(t)$ are asymptotically equivalent, too.

The asymptotic properties of estimators $\hat{\mathrm{A}}_{n}^{\mathrm{P}}(t)$ and $\hat{\mathrm{A}}_{n}^{\mathrm{CFG}}(t)$, derived in Genest and Segers (2009), are based on the empirical copula

$$
\hat{\mathrm{C}}_{n}(u, v)=\frac{1}{n} \sum_{i=1}^{n} \mathbb{I}_{\left\{\hat{U}_{i} \leq u, \hat{v}_{i} \leq v\right\}}, \forall u, v \in[0,1]
$$

More precisely, Genest and Segers' Lemma 3.1 states that, for all $t \in[0,1]$, 


\section{MARTA FERREIRA}

$$
\begin{gathered}
\sqrt{n}\left(\frac{1}{\hat{\mathrm{A}}_{n}^{\mathrm{P}}(t)}-\frac{1}{\mathrm{~A}(t)}\right)=\frac{\int_{0}^{1} \mathbb{C}_{n}\left(u^{1-t}, u^{t}\right) d u}{u} \\
\sqrt{n}\left(\log \hat{\mathrm{A}}_{n}^{\mathrm{CFG}}(t)-\log \mathrm{A}(t)\right)=\frac{\int_{0}^{1} \mathbb{C}_{n}\left(u^{1-t}, u^{t}\right) d u}{u \log u}
\end{gathered}
$$

where $\mathbb{C}_{n}$ is the empirical copula process $\sqrt{n}\left(\hat{\mathrm{C}}_{n}-\mathrm{C}\right)$. Now consider $\alpha_{n}=\sqrt{n}\left(\mathrm{C}_{n}-\mathrm{C}\right)$, with

$$
\mathrm{C}_{n}(u, v)=\frac{1}{n} \sum_{i=1}^{n} \mathbb{I}_{\left\{U_{i} \leq u, V_{i} \leq v\right\}}, \forall u, v \in[0,1]
$$

The classical theory of empirical processes states that the weak limit $\alpha$ of the process $\alpha_{n}=\sqrt{n}\left(\mathrm{C}_{n}-\mathrm{C}\right)$ is a centered Gaussian process with covariance

$$
\operatorname{cov}\left(\alpha(u, v), \alpha\left(u^{\prime}, v^{\prime}\right)\right)=\mathrm{C}\left(u \wedge v, u^{\prime} \wedge v^{\prime}\right)-\mathrm{C}(u, v) \mathrm{C}\left(u^{\prime}, v^{\prime}\right), \forall u, v, u^{\prime}, v^{\prime} \in[0,1]
$$

The weak limit $\mathbb{C}$ of the process $\mathbb{C}_{n}=\sqrt{n}\left(\hat{\mathrm{C}}_{n}-\mathrm{C}\right)$ is closely related to $\alpha$, namely,

$$
\mathbb{C}(u, v)=\alpha(u, v)-\frac{\partial \mathrm{C}(u, v)}{\partial u} \alpha(u, 1)-\frac{\partial \mathrm{C}(u, v)}{\partial v} \alpha(1, v), \forall(u, v) \in[0,1]^{2}
$$

If $\mathrm{A}$ is twice continuously differentiable on $(0,1)$ and $\sup _{\{0<t<1\}} t(1-$ t) $\mathrm{A}^{\prime \prime}(t)<\infty$, then the following weak convergence results hold, as $n \rightarrow \infty$, in the space $\mathcal{C}([0,1])$ of continuous and real-valued functions on $[0,1]$ equipped with the topology of uniform convergence:

$$
\mathbb{A}_{n}^{\mathrm{P}}=\sqrt{n}\left(\hat{\mathrm{A}}_{n}^{\mathrm{P}}(t)-\mathrm{A}(t)\right) \stackrel{w}{\rightarrow} \mathbb{A}^{\mathrm{P}}(t)=-\mathrm{A}^{2}(t) \int_{0}^{1} \frac{\mathbb{C}\left(u^{1-t}, u^{t}\right) d u}{u}
$$

and 


\section{PICKANDS DEPENDENCE FUNCTION ESTIMATION}

$$
\mathbb{A}_{n}^{\mathrm{CFG}}=\sqrt{n}\left(\hat{\mathrm{A}}_{n}^{\mathrm{CFG}}(t)-\mathrm{A}(t)\right) \stackrel{w}{\rightarrow} \mathbb{A}^{\mathrm{CFG}}(t)=\mathrm{A}(t) \int_{0}^{1} \frac{\mathbb{C}\left(u^{1-t}, u^{t}\right) d u}{u \log (u)}
$$

See Genest and Segers (2009, Theorem 3.2) and Gudendorf and Segers (2012, Theorem 1).

In the case of known margins, the results (3) and (4) hold with $\hat{\mathrm{C}}_{n}$ replaced by $\mathrm{C}_{n}$ and thus $\mathbb{C}_{n}$ replaced by $\alpha_{n}$, as well as process $\mathbb{C}$ replaced by $\alpha$ in (5) and (6). These were already proved in Segers (2007).

The new estimator can be stated for the Pickands dependence function based on Ferreira and Ferreira (2012), and will be denoted FF. Define

$$
\eta(t)=U^{1 /(1-t)} \vee V^{1 / t}
$$

with $\eta(0)=U$ and $\eta(1)=V$. By Proposition 3.1 of Ferreira and Ferreira (2012), we have

$$
\mathrm{E}(\eta(t))=1-\frac{1}{1+\mathrm{A}(t)}
$$

By an analogous reasoning used above, let

$$
\eta_{i}(t)=U_{i}^{1 /(1-t)} \vee V_{i}^{1 / t}, \quad 0<t<1
$$

with $\eta_{i}(0)=U_{i}$ and $\eta_{i}(1)=V_{i}, i=1, \ldots, n$. Thus, in the case of known margins we derive

$$
1-\frac{1}{\left(1+\mathrm{A}_{n}^{\mathrm{FF}}(t)\right)}=\frac{1}{n} \sum_{i=1}^{n} \eta_{i}(t)
$$

and, for unknown margins,

$$
1-\frac{1}{\left(1+\hat{\mathrm{A}}_{n}^{\mathrm{FF}}(t)\right)}=\frac{1}{n} \sum_{i=1}^{n} \hat{\eta}_{i}(t)
$$




\section{MARTA FERREIRA}

where $\hat{\eta}_{i}(0)=\hat{U}_{i}, \hat{\eta}_{i}(1)=\hat{V}_{i}$, and

$$
\hat{\eta}_{i}(t)=\hat{U}_{i}^{1 /(1-t)} \vee \hat{V}_{i}^{1 / t}, \quad 0<t<1
$$

with $\hat{U}_{i}$ and $\hat{V}_{i}$ as defined in (2). Because

$$
\frac{1}{n} \sum_{i=1}^{n} \hat{\eta}_{i}(0)=\frac{1}{n} \sum_{i=1}^{n} \hat{U}_{i}=\frac{1}{n} \sum_{i=1}^{n} \hat{\eta}_{i}(1)=\frac{1}{n} \sum_{i=1}^{n} \hat{V}_{i}=\frac{1}{n} \sum_{i=1}^{n} \frac{i}{n+1}=\frac{1}{2}
$$

the estimator already satisfies the constraints $\mathrm{A}_{n}^{\mathrm{FF}}(0)=\mathrm{A}_{n}^{\mathrm{FF}}(1)=1$. The following statements are direct adaptations of the results above concerning Pickands and CFG estimators.

Proposition 1: $\quad$ For all $t \in[0,1]$,

$$
\sqrt{n}\left(\frac{1}{1+\hat{\mathrm{A}}_{n}^{\mathrm{FF}}(t)}-\frac{1}{1+\mathrm{A}(t)}\right)=\int_{0}^{1} \mathbb{C}_{n}\left(u^{1-t}, u^{t}\right) d u
$$

Proof: $\quad$ Observe that

$$
\frac{1}{1+\hat{\mathrm{A}}_{n}^{\mathrm{FF}}(t)}=\frac{1}{n} \sum_{i=1}^{n} \int_{0}^{1} \mathbb{I}_{\left\{\hat{n}_{i}(t) \leq u\right\}} d u=\frac{1}{n} \sum_{i=1}^{n} \int_{0}^{1} \mathbb{I}_{\left\{\hat{U}_{i} \leq u^{1-t}, \hat{V}_{i} \leq u^{t}\right\}} d u=\int_{0}^{1} \hat{\mathrm{C}}_{n}\left(u^{1-t}, u^{t}\right) d u
$$

Proposition 2: $\quad$ If $\mathrm{A}$ is twice continuously differentiable on $(0,1)$ such that $\sup _{0<t<1} t(1-t) \mathrm{A}^{\prime \prime}(t)<\infty$, we have

$$
\mathbb{A}_{n}^{\mathrm{FF}}=\sqrt{n}\left(\hat{\mathrm{A}}_{n}^{\mathrm{FF}}(t)-\mathrm{A}(t)\right) \stackrel{w}{\rightarrow} \mathbb{A}_{n}^{\mathrm{FF}}(t)=-(\mathrm{A}(t)+1)^{2} \int_{0}^{1} \mathbb{C}\left(u^{1-t}, u^{t}\right) d u
$$

in $\mathcal{C}([0,1])$ equipped with the topology of uniform convergence.

Proof: $\quad$ Considering $u=\mathrm{e}^{-s}$ in the integral of (7), 


\section{PICKANDS DEPENDENCE FUNCTION ESTIMATION}

$$
\sqrt{n}\left(\frac{1}{1+\hat{\mathrm{A}}_{n}^{\mathrm{FF}}(t)}-\frac{1}{1+\mathrm{A}(t)}\right)=\int_{0}^{\infty} \mathbb{C}_{n}\left(\mathrm{e}^{-s(1-t)}, \mathrm{e}^{-s t}\right) \mathrm{h}(s) d s
$$

with $\mathrm{h}(s)=\mathrm{e}^{-s}$. The proof of the convergence of the integral in (9) towards $\int_{0}^{\infty} \mathbb{C}\left(\mathrm{e}^{-s(1-t)}, \mathrm{e}^{-s t}\right) \mathrm{h}(s) d s$ runs as the one of Theorem 1 in Gudendorf and Segers (2012). Now the assertion follows by applying the functional delta method (van der Vaart \& Wellner, 1996).

For the case of known margins, replace $\hat{\mathrm{C}}_{n}$ by $\mathbb{C}_{n}, \mathbb{C}_{n}$ by $\alpha_{n}$, and $\mathbb{C}$ by $\alpha$, respectively, in (7) and (8). See Gudendorf and Segers (2012) and references therein. Furthermore, Propositions 1 and 2 are extensible to the $d$-variate case for $d>2$ as stated, respectively, in Lemma 1 and Theorem 1 of Gudendorf and Segers (2012).

\section{Simulations}

Consider the most interesting case for practical purposes of unknown margins, where the performance of the new estimator is examined through simulation and compared with the corrected version of CFG and Hall and Tajvidi estimators. Specifically, 1000 random samples of size $n=100$, and of $n=1000$ were generated for each of the following models: logistic, asymmetric logistic, HüslerReiss, negative logistic, asymmetric negative logistic, bilogistic, negative bilogistic, Dirichlet, and asymmetric mixed. A description of the latter can be found in Beirlant et al. (2004).

The empirical mean integrated squared error, MISE $=\mathrm{E}\left(\int_{0}^{1}\left(\hat{\mathrm{A}}_{n}(t)-\mathrm{A}(t)\right)^{2} d t\right)$, was computed for each estimator and the obtained values are reported in Tables 1-3 (the numbers in brackets correspond to standard errors). The values of the parameters of each model were chosen in order to have the TDC $(\lambda=2(1-\mathrm{A}(0.5)))$ approximately 0.5 and the boundary cases 0 and 1, corresponding to Tables 1,2, and 3, respectively. In the unit bound case in Table 3, i.e., $\lambda \approx 1$, the considered asymmetric versions coincide with the respective symmetric models and thus omitted. Also, in the asymmetric mixed model, the largest value achieved by $\lambda$ correspond to 0.5 already reported in Table 1. Observe that the unit TDC scenario presents the smallest errors. Note the FF estimator has an overall good performance, particularly in the boundary cases of asymptotic tail independence $(\lambda \approx 0)$ and $\lambda \approx 1$ (see Tables 2 and 3 ). 


\section{MARTA FERREIRA}

Table 1. Empirical MISE values obtained for estimators CFG, HT and FF of the Pickands dependence function where the considered parameters for each model are such that $\lambda \approx 0.5$

\begin{tabular}{|c|c|c|c|c|c|c|}
\hline$n=1000$ & & CFG & & HT & & FF \\
\hline Log & $4.070 \times 10^{-5}$ & $\left(3.011 \times 10^{-6}\right)$ & $5.607 \times 10^{-5}$ & $\left(5.607 \times 10^{-6}\right)$ & $4.569 \times 10^{-5}$ & $\left(4.569 \times 10^{-6}\right)$ \\
\hline Alog & $8.383 \times 10^{-4}$ & $\left(6.200 \times 10^{-5}\right)$ & $8.403 \times 10^{-4}$ & $\left(6.199 \times 10^{-5}\right)$ & $8.496 \times 10^{-4}$ & $\left(6.268 \times 10^{-5}\right)$ \\
\hline $\mathrm{HR}$ & $3.587 \times 10^{-5}$ & $\left(3.046 \times 10^{-6}\right)$ & $4.840 \times 10^{-5}$ & $\left(4.170 \times 10^{-6}\right)$ & $3.947 \times 10^{-5}$ & $\left(3.364 \times 10^{-6}\right)$ \\
\hline Neglog & $4.181 \times 10^{-5}$ & $\left(3.306 \times 10^{-6}\right)$ & $5.560 \times 10^{-5}$ & $\left(4.444 \times 10^{-6}\right)$ & $4.609 \times 10^{-5}$ & $\left(3.669 \times 10^{-6}\right)$ \\
\hline Aneglog & $6.809 \times 10^{-5}$ & $\left(3.952 \times 10^{-6}\right)$ & $8.318 \times 10^{-5}$ & $\left(4.819 \times 10^{-6}\right)$ & $6.858 \times 10^{-5}$ & $\left(3.995 \times 10^{-6}\right)$ \\
\hline Bilog & $5.032 \times 10^{-4}$ & $\left(3.897 \times 10^{-5}\right)$ & $5.221 \times 10^{-4}$ & $\left(3.942 \times 10^{-5}\right)$ & $5.115 \times 10^{-4}$ & $\left(3.948 \times 10^{-5}\right)$ \\
\hline Negbilog & $1.063 \times 10^{-4}$ & $\left(6.854 \times 10^{-6}\right)$ & $1.200 \times 10^{-4}$ & $\left(7.558 \times 10^{-6}\right)$ & $1.123 \times 10^{-4}$ & $\left(7.204 \times 10^{-6}\right)$ \\
\hline Dir & $4.114 \times 10^{-4}$ & $\left(3.114 \times 10^{-5}\right)$ & $4.342 \times 10^{-4}$ & $\left(3.205 \times 10^{-5}\right)$ & $4.191 \times 10^{-4}$ & $\left(3.150 \times 10^{-5}\right)$ \\
\hline Amix & $4.156 \times 10^{-5}$ & $\left(3.063 \times 10^{-6}\right)$ & $5.621 \times 10^{-5}$ & $\left(4.319 \times 10^{-6}\right)$ & $4.604 \times 10^{-5}$ & $\left(3.401 \times 10^{-6}\right)$ \\
\hline$n=100$ & & CFG & & HT & & FF \\
\hline Log & $2.890 \times 10^{-4}$ & $\left(2.861 \times 10^{-6}\right)$ & $4.181 \times 10^{-4}$ & $\left(4.140 \times 10^{-6}\right)$ & $3.656 \times 10^{-4}$ & $\left(3.323 \times 10^{-6}\right)$ \\
\hline Alog & $1.289 \times 10^{-3}$ & $\left(7.866 \times 10^{-5}\right)$ & $1.436 \times 10^{-3}$ & $\left(8.335 \times 10^{-5}\right)$ & $1.403 \times 10^{-3}$ & $\left(8.386 \times 10^{-5}\right)$ \\
\hline $\mathrm{HR}$ & $3.544 \times 10^{-4}$ & $\left(3.035 \times 10^{-5}\right)$ & $4.595 \times 10^{-4}$ & $\left(4.011 \times 10^{-5}\right)$ & $4.043 \times 10^{-4}$ & $\left(3.385 \times 10^{-5}\right)$ \\
\hline Neglog & $3.948 \times 10^{-4}$ & $\left(3.246 \times 10^{-5}\right)$ & $5.368 \times 10^{-4}$ & $\left(4.482 \times 10^{-5}\right)$ & $4.584 \times 10^{-4}$ & $\left(3.713 \times 10^{-5}\right)$ \\
\hline Aneglog & $6.150 \times 10^{-4}$ & $\left(3.735 \times 10^{-5}\right)$ & $7.542 \times 10^{-4}$ & $\left(4.435 \times 10^{-5}\right)$ & $6.787 \times 10^{-4}$ & $\left(4.027 \times 10^{-5}\right)$ \\
\hline Bilog & $8.055 \times 10^{-4}$ & $\left(5.198 \times 10^{-5}\right)$ & $9.542 \times 10^{-4}$ & $\left(6.003 \times 10^{-5}\right)$ & $8.872 \times 10^{-4}$ & $\left(5.600 \times 10^{-5}\right)$ \\
\hline Negbilog & $4.231 \times 10^{-4}$ & $\left(3.147 \times 10^{-5}\right)$ & $5.505 \times 10^{-4}$ & $\left(4.182 \times 10^{-5}\right)$ & $4.786 \times 10^{-4}$ & $\left(3.489 \times 10^{-5}\right)$ \\
\hline Dir & $7.399 \times 10^{-4}$ & $\left(4.869 \times 10^{-5}\right)$ & $8.956 \times 10^{-4}$ & $\left(5.916 \times 10^{-5}\right)$ & $8.117 \times 10^{-4}$ & $\left(5.214 \times 10^{-5}\right)$ \\
\hline Amix & $4.249 \times 10^{-4}$ & $\left(3.462 \times 10^{-5}\right)$ & $5.617 \times 10^{-4}$ & $\left(4.730 \times 10^{-5}\right)$ & $4.748 \times 10^{-4}$ & $\left(3.752 \times 10^{-5}\right)$ \\
\hline
\end{tabular}

Note: Numbers in brackets correspond to standard errors

Table 2. Empirical MISE values obtained for estimators CFG, HT and FF of the Pickands dependence function, in the case of asymptotic tail independence $(\lambda \approx 0)$

\begin{tabular}{|c|c|c|c|c|c|c|}
\hline$n=1000$ & & CFG & & HT & & FF \\
\hline Log & $1.020 \times 10^{-4}$ & $\left(5.090 \times 10^{-6}\right)$ & $1.997 \times 10^{-4}$ & $\left(1.017 \times 10^{-5}\right)$ & $7.133 \times 10^{-5}$ & $\left(3.616 \times 10^{-6}\right)$ \\
\hline Alog & $9.932 \times 10^{-5}$ & $\left(4.885 \times 10^{-6}\right)$ & $2.103 \times 10^{-4}$ & $\left(1.042 \times 10^{-5}\right)$ & $6.230 \times 10^{-5}$ & $\left(3.007 \times 10^{-6}\right)$ \\
\hline $\mathrm{HR}$ & $1.054 \times 10^{-4}$ & $\left(5.203 \times 10^{-6}\right)$ & $2.212 \times 10^{-4}$ & $\left(1.068 \times 10^{-5}\right)$ & $7.121 \times 10^{-5}$ & $\left(3.499 \times 10^{-6}\right)$ \\
\hline Neglog & $1.021 \times 10^{-4}$ & $\left(5.161 \times 10^{-6}\right)$ & $2.052 \times 10^{-4}$ & $\left(1.065 \times 10^{-5}\right)$ & $6.792 \times 10^{-5}$ & $\left(3.502 \times 10^{-6}\right)$ \\
\hline Aneglog & $1.032 \times 10^{-4}$ & $\left(5.171 \times 10^{-6}\right)$ & $2.101 \times 10^{-4}$ & $\left(1.081 \times 10^{-5}\right)$ & $6.890 \times 10^{-5}$ & $\left(3.468 \times 10^{-6}\right)$ \\
\hline Bilog & $1.025 \times 10^{-4}$ & $\left(5.413 \times 10^{-5}\right)$ & $2.093 \times 10^{-4}$ & $\left(1.145 \times 10^{-5}\right)$ & $7.438 \times 10^{-5}$ & $\left(4.023 \times 10^{-6}\right)$ \\
\hline Negbilog & $1.042 \times 10^{-4}$ & $\left(5.279 \times 10^{-6}\right)$ & $2.142 \times 10^{-4}$ & $\left(1.123 \times 10^{-5}\right)$ & $7.067 \times 10^{-5}$ & $\left(3.565 \times 10^{-6}\right)$ \\
\hline Dir & $1.022 \times 10^{-4}$ & $\left(5.162 \times 10^{-5}\right)$ & $2.072 \times 10^{-4}$ & $\left(1.060 \times 10^{-5}\right)$ & $7.737 \times 10^{-5}$ & $\left(4.080 \times 10^{-6}\right)$ \\
\hline Amix & $1.054 \times 10^{-4}$ & $\left(5.248 \times 10^{-6}\right)$ & $2.100 \times 10^{-4}$ & $\left(1.054 \times 10^{-5}\right)$ & $7.307 \times 10^{-5}$ & $\left(3.698 \times 10^{-6}\right)$ \\
\hline
\end{tabular}

Note: Numbers in brackets correspond to standard errors 


\section{PICKANDS DEPENDENCE FUNCTION ESTIMATION}

Table 2, continued.

\begin{tabular}{|c|c|c|c|c|c|c|}
\hline$n=100$ & & CFG & & HT & & FF \\
\hline Log & $1.404 \times 10^{-3}$ & $\left(6.483 \times 10^{-5}\right)$ & $2.232 \times 10^{-3}$ & $\left(1.120 \times 10^{-4}\right)$ & $9.676 \times 10^{-4}$ & $\left(4.309 \times 10^{-5}\right)$ \\
\hline Alog & $1.349 \times 10^{-3}$ & $\left(6.389 \times 10^{-5}\right)$ & $2.165 \times 10^{-3}$ & $\left(1.107 \times 10^{-4}\right)$ & $9.250 \times 10^{-4}$ & $\left(4.308 \times 10^{-5}\right)$ \\
\hline HR & $1.350 \times 10^{-3}$ & $\left(6.161 \times 10^{-5}\right)$ & $2.121 \times 10^{-3}$ & $\left(1.096 \times 10^{-4}\right)$ & $9.128 \times 10^{-4}$ & $\left(3.889 \times 10^{-5}\right)$ \\
\hline Neglog & $1.344 \times 10^{-3}$ & $\left(6.274 \times 10^{-5}\right)$ & $2.181 \times 10^{-3}$ & $\left(1.128 \times 10^{-4}\right)$ & $8.938 \times 10^{-4}$ & $\left(3.966 \times 10^{-5}\right)$ \\
\hline Aneglog & $1.441 \times 10^{-3}$ & $\left(6.655 \times 10^{-5}\right)$ & $2.141 \times 10^{-3}$ & $\left(1.064 \times 10^{-4}\right)$ & $1.001 \times 10^{-4}$ & $\left(4.488 \times 10^{-5}\right)$ \\
\hline Bilog & $1.339 \times 10^{-3}$ & $\left(6.351 \times 10^{-5}\right)$ & $2.123 \times 10^{-3}$ & $\left(1.052 \times 10^{-4}\right)$ & $9.496 \times 10^{-4}$ & $\left(4.462 \times 10^{-5}\right)$ \\
\hline Negbilog & $1.236 \times 10^{-3}$ & $\left(5.570 \times 10^{-5}\right)$ & $1.989 \times 10^{-3}$ & $\left(1.035 \times 10^{-4}\right)$ & $8.316 \times 10^{-4}$ & $\left(3.542 \times 10^{-5}\right)$ \\
\hline Dir & $1.345 \times 10^{-3}$ & $\left(6.343 \times 10^{-5}\right)$ & $2.087 \times 10^{-3}$ & $\left(1.047 \times 10^{-4}\right)$ & $9.509 \times 10^{-4}$ & $\left(4.345 \times 10^{-5}\right)$ \\
\hline Amix & $1.409 \times 10^{-3}$ & $\left(6.608 \times 10^{-5}\right)$ & $2.190 \times 10^{-3}$ & $\left(1.139 \times 10^{-4}\right)$ & $9.676 \times 10^{-4}$ & $\left(4.348 \times 10^{-5}\right)$ \\
\hline
\end{tabular}

Note: Numbers in brackets correspond to standard errors

Table 3. Empirical MISE values obtained for estimators CFG, HT and FF of the Pickands dependence function, in the case $\lambda \approx 1$

\begin{tabular}{|c|c|c|c|c|c|c|}
\hline$n=100$ & & CFG & & HT & & FF \\
\hline Log & $3.874 \times 10^{-9}$ & $\left(2.394 \times 10^{-9}\right)$ & $3.539 \times 10^{-9}$ & $\left(2.262 \times 10^{-9}\right)$ & $6.118 \times 10^{-10}$ & $\left(3.926 \times 10^{-10}\right)$ \\
\hline $\mathrm{HR}$ & $4.930 \times 10^{-10}$ & $\left(2.935 \times 10^{-9}\right)$ & $4.413 \times 10^{-9}$ & $\left(2.768 \times 10^{-9}\right)$ & $5.571 \times 10^{-10}$ & $\left(3.625 \times 10^{-10}\right)$ \\
\hline Neglog & $4.001 \times 10^{-9}$ & $\left(2.451 \times 10^{-9}\right)$ & $3.709 \times 10^{-9}$ & $\left(2.378 \times 10^{-9}\right)$ & $5.826 \times 10^{-10}$ & $\left(3.753 \times 10^{-10}\right)$ \\
\hline Bilog & $3.913 \times 10^{-9}$ & $\left(2.400 \times 10^{-10}\right)$ & $3.610 \times 10^{-9}$ & $\left(2.312 \times 10^{-9}\right)$ & $6.220 \times 10^{-10}$ & $\left(4.000 \times 10^{-10}\right)$ \\
\hline Negbilog & $4.131 \times 10^{-9}$ & $\left(2.464 \times 10^{-9}\right)$ & $3.517 \times 10^{-9}$ & $\left(2.276 \times 10^{-9}\right)$ & $5.985 \times 10^{-10}$ & $\left(2.869 \times 10^{-10}\right)$ \\
\hline Dir & $2.890 \times 10^{-8}$ & $\left(2.612 \times 10^{-8}\right)$ & $2.154 \times 10^{-7}$ & $\left(3.900 \times 10^{-8}\right)$ & $8.186 \times 10^{-8}$ & $\left(2.721 \times 10^{-8}\right)$ \\
\hline$n=100$ & & CFG & & HT & & $\mathrm{FF}$ \\
\hline $\log$ & $1.530 \times 10^{-7}$ & $\left(7.468 \times 10^{-8}\right)$ & $1.352 \times 10^{-7}$ & $\left(7.366 \times 10^{-8}\right)$ & $1.342 \times 10^{-8}$ & $\left(1.062 \times 10^{-8}\right)$ \\
\hline $\mathrm{HR}$ & $1.872 \times 10^{-7}$ & $\left(9.113 \times 10^{-8}\right)$ & $1.627 \times 10^{-7}$ & $\left(8.760 \times 10^{-8}\right)$ & $1.903 \times 10^{-8}$ & $\left(1.487 \times 10^{-8}\right)$ \\
\hline Neglog & $1.492 \times 10^{-7}$ & $\left(7.352 \times 10^{-8}\right)$ & $1.348 \times 10^{-7}$ & $\left(7.360 \times 10^{-8}\right)$ & $1.279 \times 10^{-8}$ & $\left(1.018 \times 10^{-8}\right)$ \\
\hline Bilog & $1.516 \times 10^{-7}$ & $\left(7.519 \times 10^{-8}\right)$ & $1.342 \times 10^{-7}$ & $\left(7.256 \times 10^{-8}\right)$ & $1.606 \times 10^{-8}$ & $\left(1.035 \times 10^{-8}\right)$ \\
\hline Negbilog & $1.519 \times 10^{-7}$ & $\left(7.513 \times 10^{-8}\right)$ & $1.361 \times 10^{-7}$ & $\left(7.457 \times 10^{-8}\right)$ & $1.265 \times 10^{-8}$ & $\left(1.003 \times 10^{-8}\right)$ \\
\hline Dir & $2.074 \times 10^{-6}$ & $\left(5.517 \times 10^{-7}\right)$ & $2.033 \times 10^{-6}$ & $\left(6.234 \times 10^{-7}\right)$ & $1.250 \times 10^{-6}$ & $\left(4.285 \times 10^{-7}\right)$ \\
\hline
\end{tabular}

Note: Numbers in brackets correspond to standard errors

\section{Conclusion}

A new estimator for the Pickands dependence function, an important map in generating extreme value copulas, was presented. It was found via simulation that it may be used as an alternative to the well-known CFG estimator, especially in the limiting situation of asymptotic tail independence. Thus, it may have a promising performance in testing independence, a crucial issue in statistics of extremes. 


\section{MARTA FERREIRA}

\section{Acknowledgements}

The author wishes to thank the reviewers for their careful reading and relevant comments that have improved this work. This research was financed by Portuguese Funds through FCT - Fundação para a Ciência e a Tecnologia within

the Projects UID/MAT/00013/2013, UID/MAT/00006/2013, and UID/Multi/04621/2013.

\section{References}

Abdous, B., \& Ghoudi, K. (2005). Non-parametric estimators of multivariate extreme dependence functions. Journal of Nonparametric Statistics, 17(8), 915-935. doi: 10.1080/10485250500336379

Beirlant, J., Goegebeur, Y., Segers, J., \& Teugels, J. (2004). Statistics of extremes: Theory and applications. Chichester, UK: Wiley. doi: 10.1002/0470012382

Capéraà, P., Fougères, A.-L., \& Genest, C. (1997). A nonparametric estimation procedure for bivariate extreme value copulas. Biometrika, 84(3), 567577. doi: 10.1093/biomet/84.3.567

Cebrian, A., Denuit, M., \& Lambert, P. (2003). Analysis of bivariate tail dependence using extreme values copulas: An application to the SOA medical large claims database. Belgian Actuarial Bulletin, 3(1), 33-41.

Coles, S. G., Heffernan, J., \& Tawn, J. (1999). Dependence measures for extreme value analyses. Extremes, 2(4), 339-365. doi: 10.1023/a:1009963131610

Deheuvels, P. (1984). Probabilistic aspects of multivariate extremes. In J. T. de Oliveira (Ed.), Statistical extremes and applications (pp. 117-130). Boston, MA: D. Reidel Pub. Co. doi: 10.1007/978-94-017-3069-3_9

Deheuvels, P. (1991). On the limiting behavior of the Pickands estimator for bivariate extreme-value distributions. Statistics \& Probability Letters, 12(5), 429439. doi: 10.1016/0167-7152(91)90032-m

Ferreira, H. \& Ferreira, M. (2012). On extremal dependence of block vectors. Kybernetika, 48(5), 988-1006. Retrieved from http://www.kybernetika.cz/content/2012/5/988

Frees, E. W., \& Valdez, E. A. (1998). Understanding relationships using copulas. North American Actuarial Journal, 2(1), 1-25. doi: 10.1080/10920277.1998.10595667 


\section{PICKANDS DEPENDENCE FUNCTION ESTIMATION}

Genest, C., \& Segers, J. (2009). Rank-based inference for bivariate extremevalue copulas. The Annals of Statistics, 37(5B), 2990-3022 . doi: 10.1214/08$\operatorname{aos} 672$

Ghoudi, K., Khoudraji, A., \& Rivest, L.-P. (1998). Propriétés statistiques des copules de valeurs extrêmes bidimensionnelles. The Canadian Journal of Statistics, 26(1), 187-197. doi: 10.2307/3315683

Gudendorf, G., \& Segers, J. (2011). Nonparametric estimation of an extreme-value copula in arbitrary dimensions. Journal of Multivariate Analysis, 102(1), 37-47. doi: 10.1016/j.jmva.2010.07.011

Gudendorf, G., \& Segers, J. (2012). Nonparametric estimation of multivariate extreme-value copulas. Journal of Statistical Planning and Inference, 142(12), 3073-3085. doi: 10.1016/j.jspi.2012.05.007

Hall, P., \& Tajvidi, N. (2000). Distribution and dependence-function estimation for bivariate extreme-value distributions. Bernoulli, 6(5), 835-844. doi: $10.2307 / 3318758$

Hsing, T. (1989). Extreme value theory for multivariate stationary sequences. Journal of Multivariate Analysis, 29(2), 274-291. doi: 10.1016/0047259x(89)90028-6

Jiménez, J. R., Villa-Diharce, E., \& Flores, M. (2001). Nonparametric estimation of the dependence function in bivariate extreme value distributions. Journal of Multivariate Analysis, 76(2), 159-191. doi: 10.1006/jmva.2000.1931

McNeil, A. J., Frey, R., \& Embrechts, P. (2005). Quantitative risk management: Concepts, techniques and tools. Princeton, NJ: Princeton University Press.

Pickands, J. (1981). Multivariate extreme value distributions. In Proceedings of the 43rd Session of the International Statistical Institute (Vol. 2, pp. 859-878). Buenos Aires, Brazil: International Statistical Institute.

Salvadori, G., De Michele, C., Kottegoda, N. T., \& Rosso, R. (2007). Extremes in nature: An approach using copulas. Dordrecht, Netherlands: Springer. doi: 10.1007/1-4020-4415-1

Segers, J. (2007). Nonparametric inference for bivariate extreme-value copulas. In M. Ahsanullah \& S. N. U. A. Kirmani (Eds.), Topics in extreme values (pp. 185-207). New York, NY: Nova Science Publishers.

Sibuya, M. (1960). Bivariate extreme statistics. Annals of the Institute of Statistical Mathematics, 11(2), 195-210. doi: 10.1007/bf01682329 


\section{MARTA FERREIRA}

Sklar, M. (1959). Fonctions de répartition à $\mathrm{n}$ dimensions et leurs marges. Publications de l'Institut de Statistique de l'Université de Paris, 8, 229-231.

Tawn, J. A. (1988). Bivariate extreme value theory: Models and estimation. Biometrika, 75(3), 397-415. doi: 10.1093/biomet/75.3.397

van der Vaart, A. W., \& Wellner, J. A. (1996). Weak convergence and empirical processes: With applications to statistics. New York, NY: Springer. doi: 10.1007/978-1-4757-2545-2

Zhang, D., Wells, M. T., \& Peng, L. (2008). Nonparametric estimation of the dependence function for a multivariate extreme value distribution. Journal of Multivariate Analysis, 99(4), 577-588. doi: 10.1016/j.jmva.2006.09.011 